\title{
A Novel INDI based Guidance Law for Fixed Wing Aircrafts: Derivation and Application
}

This paper was downloaded from TechRxiv (https://www.techrxiv.org).

LICENSE

CC BY 4.0

SUBMISSION DATE / POSTED DATE

$09-01-2022$ / 12-01-2022

\section{CITATION}

Li, Mengtang; Ding, Beichen (2022): A Novel INDI based Guidance Law for Fixed Wing Aircrafts: Derivation and Application. TechRxiv. Preprint. https://doi.org/10.36227/techrxiv.18093254.v1

$\mathrm{DOI}$

10.36227/techrxiv.18093254.v1 


\section{Title}

A Novel INDI based Guidance Law for Fixed Wing Aircrafts: Derivation and Application

\section{Author}

Beichen Ding, Ph.D., dingbch@mail.sysu.edu.cn

Mengtang Li*, Ph.D., AIAA Member, limt29@mail.sysu.edu.cn

\section{Institution}

School of Intelligent Systems Engineering, Sun Yat-Sen University, Guangzhou, Guangdong, China, 510000. 


\begin{abstract}
This paper presents a novel guidance law for a fixed wing aircraft to capture and track a straight reference line. Trajectory error dynamics and transformations of coordinate systems were established first, and the guidance laws were derived based on the principle of incremental nonlinear dynamic inversion (INDI) and conventional PD methods. The proposed formula is more concise and the physical concept behind is clearer through the utilization of mathematical operations in the derivations. Conventional longitudinal and lateral commands are generated from the outer loop, allowing modularly working with various inner loop control methods without tedious parameter tuning. Finally, the proposed scheme was applied to the guidance law design of LOC/GLS mode, LOC/ALT mode and PHI/ALT mode for an aircraft, and the preliminary simulations were conducted. The 6 degree of freedom (DoF) simulation results showed that the guidance laws designed according to the proposed scheme can achieve precise trajectory tracking and demonstrate satisfactory dynamic characteristics and robustness.
\end{abstract}

Key words: Nonlinear Dynamic Inversion (NDI), Incremental Nonlinear Dynamic Inversion (INDI), Localizer (LOC), Glideslope (GLS), Altitude (ALT). 
Nomenclature

\begin{tabular}{|c|c|c|}
\hline Symbol & Description & property \\
\hline$\{B\}$ & Body frame & Frame \\
\hline$\{\bar{B}\}$ & Intermediate Body Frame & Frame \\
\hline$\{E\}$ & Earth-Centered Earth Fixed frame & Frame \\
\hline$\{I\}$ & Earth-Centered Inertial frame & Frame \\
\hline$\{K\}$ & Kinematic frame and its origin & Frame \\
\hline$\{N\}$ & navigation frame and its origin & Frame \\
\hline$\{0\}$ & North-East-Down frame & Frame \\
\hline$\{T\}$ & Reference trajectory frame and its origin & Frame \\
\hline $\mathrm{F}$ & Projection of G onto the reference trajectory & point \\
\hline G & Center of mass of an aircraft & point \\
\hline g & Gravity & Scalar constant, predefined \\
\hline$K_{P Y}, K_{P Z}$ & Proportional gains for $\mathrm{y}$ and $\mathrm{z}$ channels & Scalar constant, predefined \\
\hline$K_{D Y}, K_{D Z}$ & Derivative gains for $\mathrm{y}$ and $\mathrm{z}$ channels & Scalar constant, predefined \\
\hline$\left(\boldsymbol{N}_{G}\right)_{B}$ & aircraft's load factor expressed in frame $\{B\}$ & Vector variable, measured \\
\hline$N_{z c m d}$ & normal load factor command & Scalar variable, calculated \\
\hline $\mathrm{s}$ & displacement vector & Vector variable, calculated \\
\hline$P_{S}$ & roll rate around an aircraft's stability axis & Scalar variable, calculated \\
\hline$P_{\text {scmd }}$ & stability-axis roll rate command & Scalar variable, calculated \\
\hline$P$ & Aircraft's roll rate & Scalar variable, measured \\
\hline$Q$ & Aircraft's pitch rate & Scalar variable, measured \\
\hline$R$ & Aircraft's yaw rate & Scalar variable, measured \\
\hline$V_{G}$ & Aircraft's speed long $\mathrm{x}$-axis of frame $\{\mathrm{K}\}$ & Scalar variable, measured \\
\hline$\Delta x_{T}$ & Forward trajectory error & Scalar variable, ignored \\
\hline$\Delta y_{T}, \Delta \dot{y}_{T}$ & $\begin{array}{l}\text { lateral trajectory error and corresponding time } \\
\text { derivative }\end{array}$ & Scalar variable, calculated \\
\hline$\Delta z_{T}, \Delta \dot{z}_{T}$ & $\begin{array}{l}\text { vertical trajectory error and corresponding time } \\
\text { derivative }\end{array}$ & Scalar variable, calculated \\
\hline$\Delta \ddot{y}_{T, \text { des }}$ & desired lateral and vertical accelerations & Scalar variable, calculated \\
\hline \multicolumn{3}{|l|}{$\Delta \ddot{Z}_{T, d e s}$} \\
\hline$\alpha$ & Aircraft's angle of attack & Vector variable, measured \\
\hline$\phi$ & Aircraft's roll angle & Vector variable, measured \\
\hline$\theta$ & Aircraft's pitch angle & Vector variable, measured \\
\hline$\psi$ & Aircraft's yaw angle & Vector variable, measured \\
\hline$\chi_{k}$ & aircraft's flight path azimuth angle & Scalar variable, measured \\
\hline$\gamma_{k}$ & aircraft's flight path inclination angle & Scalar variable, measured \\
\hline$\chi_{T}$ & Reference trajectory azimuth angle & Scalar constant, predefined \\
\hline$\gamma_{T}$ & Reference trajectory inclination angle & Scalar constant, predefined \\
\hline$\omega_{n y}, \omega_{n z}$ & $\begin{array}{l}\text { Bandwidth of lateral and vertical trajectory error } \\
\text { regulators }\end{array}$ & Scalar constant, predefined \\
\hline$\zeta_{y}, \zeta_{z}$ & $\begin{array}{l}\text { damping ratio of lateral and vertical trajectory error } \\
\text { regulators }\end{array}$ & Scalar constant, predefined \\
\hline
\end{tabular}




\section{Introduction}

Contemporary flight vehicles including fighter jets and fixed wing unmanned aerial vehicles (UAVs) become more advanced and more intelligently automated. Autopilot guidance law for trajectory or path capturing and tracking is a main research subject with promising applications such as automatic landing on a stationary/moving/floating runway, cursing monitoring and surveillance. The widely used classic proportional-integral-derivative (PID) control utilized in trajectory guidance performs unsatisfactorily poor when the flight vehicle operates in a highly nonlinear stage or under large disturbances [1] and it is time consuming to obtain fine gain scheduling for versatile flight conditions [2]. Flight stability is only guaranteed in the vicinity of a reference path for classical linear PID guidance law [3]. Further, different flight platforms normally demand redesigning guidance controllers to achieve specific applications [4]. Hence, a modular designed guidance control method with a generic structure is preferably desired to be capable of working with various low level inner loop controls on versatile flight platforms.

Research has been done to seek a better guidance design method to resolve previously mentioned shortcomings such as custom controller design, parameter tuning, low design efficiency and unsatisfactory control performance brought by the wildly used classical methods. The guidance methods for three-dimensional reference trajectory capturing and following can be categorized into three classes: error dynamic based, virtual potential field based, and virtual target following based approaches [3]. Examples of controlling tracking error dynamics [5,6] demonstrate good flight performance and global stability. Nonetheless, tracking accuracy is limited by not considering geometric information of a trajectory or flight vehicle's inherent information. Virtual potential field as its name has suggested utilizing a potential field to drag a flight vehicle to achieve high tracking accuracy and global stability with simpler control laws $[7,8]$. However, this method does not take the aircraft's dynamics into account and therefore only work for a certain type of flight vehicles, for instance a quadrotor drone, and specific types of reference paths. The virtual target method generates a moving reference point on the desired trajectory for the aircraft to follow $[9,10]$. Cho et al [11] proposed a nonlinear differential geometric path-following guidance law which aligns the velocity of the vehicle with the look-ahead vector and generates a normal acceleration command required for exact path-following. Satisfied performance is mathematically and simulationally verified, but the generated acceleration commend still needs further "translations" to allow an aircraft to receive and to therefore allocate various actuators to perform necessary maneuvers.

Nonlinear dynamic inversion (NDI) method [12] linearizes the input-output relation to allow usage of common linear control theory to nonlinear systems, and flight qualities are optimized from points of PID methodology to space of NDI methodology. Through employment of NDI method, Xie et. al. [13] designed three loops based on NDI principles using first order reference model to control flight path angle and airspeed, attitude angle and angular speed, respectively, to ultimately achieve an inertial trajectory tracking. Oliveira et. al. [14] proposed a similar control method to track ground target. Schatz and Holzapfel [4] proposed an NDI based modular second order 
error dynamics trajectory controller through the fundamental principles of motion equations and completed flight test verification on UAVs. Noteworthily in Schatz's method, only the normal load guidance command is derived and sent to the inner loop for longitudinal control. No other alternative command option is provided if the normal load factor guidance command does not meet performance requirements. The derivation process is too bulky and burdensome, leaving room for simplification and improvement. Lastly, though NDI method can mathematically eliminate all the nonlinearities and linearize the system, it needs heavy and accurate modelling and is sensitive to any model inaccuracy [15]. Compared with classical NDI, the incremental NDI (INDI) [16] takes advantages of sensor measurements to account for a large portion of the accurate modeling, decreasing model dependency and increasing robustness. Smeur et. al. [17] proposed an adaptive INDI method for attitude control of quadrotor micro air vehicles (MAVs). The control effectiveness matrices could be adapted online and also scaled to other platforms. Since the controlled target is quadrotors and therefore the kinematics is simpler compared with fixed wing aircrafts, the trajectory capturing and tracking control was not studied.

Motivated by the aforementioned demands for current fixed wing aircrafts, a novel modular guidance control method which utilizes INDI principles of second order nonlinear error dynamics, which considers the aircraft's intrinsic kinematics, is proposed in this paper to allow the community to apply it onto various platforms, mainly fixed wing jets or UAVs, without concerning about the aforementioned issues. This paper is organized as follows. Section II presents the trajectory guidance research object with a block diagram showing the principle of the proposed guidance and control system. Specific contents, namely the trajectory error calculation, regulation, and outer loop control, are further introduced in the following Section III, IV, and V, respectively. Section VI establishes simulation applications, where the proposed guidance method is validated and evaluated. Discussions and conclusions are drawn in the last Section VII. For the readers' convenience, handy and critical coordinate systems and related transformation matrices are included in the Appendix Section.

\section{Trajectory Guidance Law Design}

The reference trajectories considered are a group of straight lines which could be used for instance aircraft automatic approach and landing. Each segment of straight lines origins at a point $S$ and orientates with two reference trajectory angles: reference trajectory azimuth angle $\chi_{T}$ and reference trajectory inclination angle $\gamma_{T}$, as shown in Fig.1. The frame $\left\{N x_{N} y_{N} z_{N}\right\}$ is navigation frame $\{N\}$, whose origin $N$ is a point fixed on Earth. The frame $\left\{T x_{T} y_{T} z_{T}\right\}$ is reference trajectory frame $\{T\}$ with its origin $T$ coincides with $S$. Details of frame $\{N\}$ and $\{T\}$ are introduced in Appendix 1 and 2 . 


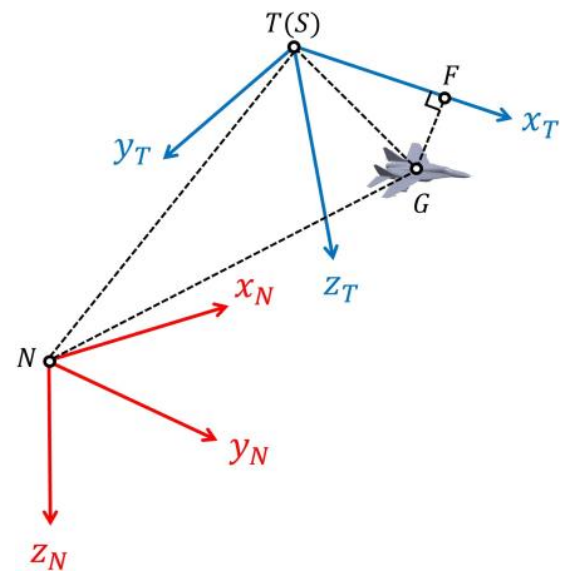

Fig. 1 Reference trajectory of one segment of straight lines

The schematic of the proposed trajectory guidance and control system is depicted in Fig.2, where $\Delta y_{T}, \Delta z_{T}, \Delta \dot{y}_{T}, \Delta \dot{z}_{T}$ are lateral and vertical trajectory errors and corresponding derivatives, respectively; $\Delta \ddot{y}_{T, \text { des }}, \Delta \ddot{z}_{T, \text { des }}$ are desired lateral and vertical accelerations of an aircraft under frame $\{T\} ; N_{z c m d}$ and $P_{\text {scmd }}$ are normal load factor command and stability-axis roll rate command, respectively. Main components of the outer loop include trajectory error and derivative computation, trajectory error regulator, and outer loop INDI guidance law. The inner loop handles aircraft stability and control augmentation. It receives $N_{z c m d}$ and $P_{\text {scmd }}$, two conventional commands, and generates necessary actuator deflection commands to an aircraft. Since different flight platforms have various inner loop control methods, the inner loop control is not the main focus of the paper and is assumed to have satisfied control law: normal load factor control law is used for the longitudinal channel, stability-axis roll rate control law is used for the lateral channel, and a blended sideslip angle and lateral load factor control law is used for yaw channel.

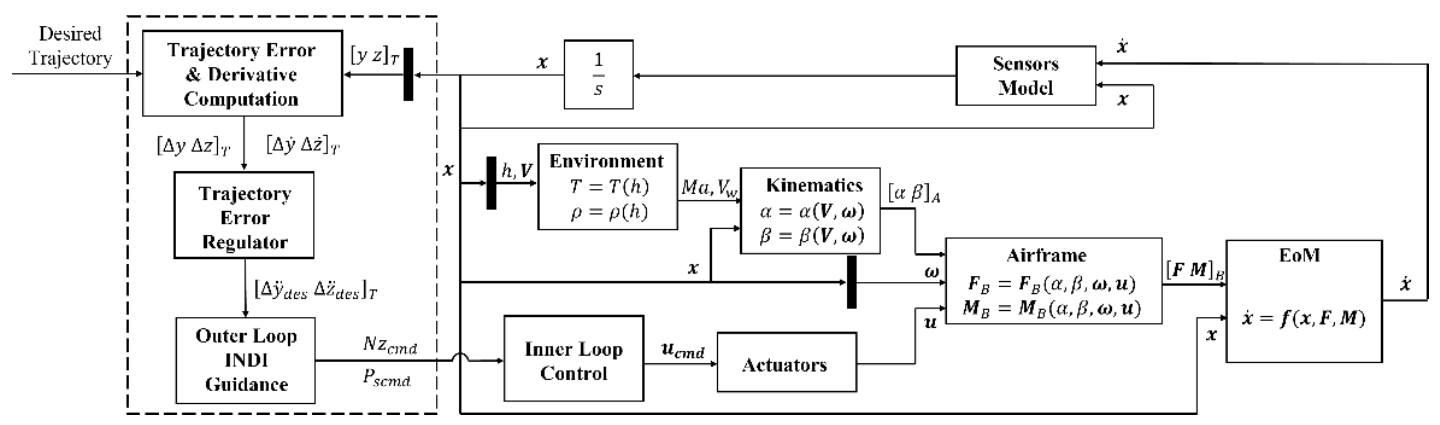

Fig. 2 Block diagram of trajectory guidance and control system

The subsequent sections derive the aforementioned blocks shown in Fig.2. Mathematical derivations in this paper are calculated under the following conventional assumptions [4]:

1. The earth is flat, thus there is no rotation between the Earth-Centered Inertial frame $\{I\}$ and the Earth-Centered Earth Fixed frame $\{E\}$

2. The earth is not rotating, thus there is no rotation between the North-EastDown frame $\{O\}$ and frame $\{E\}$ 
3. The considered aircraft is a rigid body and the mass of it does not vary due to fuel usage.

4. Center of Gravity (CoG) of the considered aircraft is chosen as the reference point (point $G$ shown in Fig.1).

\section{Trajectory Error and Derivative Computation}

The projection of the $\mathrm{CoG}$ of aircraft onto the reference trajectory (a straight line) is denoted as $F$. Vector $\boldsymbol{s}_{F G}$ (from $\mathrm{F}$ to $\mathrm{G}$ ) is defined as trajectory error. The purpose of a trajectory guidance law is to control an aircraft to eliminate $\boldsymbol{s}_{F G}$ smoothly and fast. The trajectory guidance control block, in the outer loop, sends appropriate commands to the inner loop to achieve the guidance task.

In the vector triangle $N T G$, there exists the follow geometric relation:

$$
\boldsymbol{s}_{N G}=\boldsymbol{s}_{N T}+\boldsymbol{s}_{T G}
$$

The displacement vector $\boldsymbol{s}_{T G}$ of point $G$ relative to point $T$ under frame $\{T\}$ can be calculated as follows:

$$
\left(\boldsymbol{s}_{T G}\right)_{T}=\boldsymbol{M}_{T N}\left[\left(\boldsymbol{s}_{N G}\right)_{N}-\left(\boldsymbol{s}_{N T}\right)_{N}\right]
$$

where $\boldsymbol{M}_{T N}$ is the transformation matrix from frame $\{N\}$ to frame $\{T\}$ and its detailed calculation is shown in Appendix-2; displacement vector of point $G$ and $T$ relative to point $N$ can be measured more readily under frame $\{N\}$ and are denoted as $\left(\boldsymbol{s}_{N G}\right)_{N},\left(\boldsymbol{s}_{N T}\right)_{N}$ respectively

The trajectory error vector $\left(\boldsymbol{s}_{F G}\right)_{T}$ under frame $\{T\}$ is then

$$
\begin{aligned}
\left(\boldsymbol{s}_{F G}\right)_{T} & =\left[\begin{array}{lll}
\Delta x_{T} & \Delta y_{T} & \Delta z_{T}
\end{array}\right]^{T} \\
& =\left(\boldsymbol{R}_{F G T G}\right)_{T}\left(\boldsymbol{s}_{T G}\right)_{T}
\end{aligned}
$$

where $\left(\boldsymbol{R}_{F G T G}\right)_{T}$ is the projection tensor for $\boldsymbol{s}_{T G}$ onto $\boldsymbol{s}_{F G}$ under frame $\{T\}$, which simply selects y and z columns and ignores x column [18]:

$$
\left(\boldsymbol{R}_{F G T G}\right)_{T}=\left[\begin{array}{lll}
0 & & \\
& 1 & \\
& & 1
\end{array}\right]
$$

The differential velocity of $\boldsymbol{s}_{F G}$ under frame $\{T\}$ can be obtained by taking time derivative of Eq. (3) on both sides. The time derivative of $\boldsymbol{s}_{T G}$ under frame $\{T\}$ can be calculated more easily by measuring the aircraft's velocity $\left(\left(\boldsymbol{v}_{G}\right)_{k}=\right.$ $\left[\begin{array}{lll}V_{G} & 0 & 0\end{array}\right]^{T}$ ) relative to the Earth expressed under kinematic frame $\{K\}$ and then transforming to be expressed under frame $\{T\}$. 


$$
\begin{aligned}
\left(v_{F G}\right)_{T} & =\left[\begin{array}{lll}
\Delta \dot{x}_{T} & \Delta \dot{y}_{T} & \Delta \dot{z}_{T}
\end{array}\right]^{T} \\
& =\left(\boldsymbol{R}_{F G T G}\right)_{T}\left(\boldsymbol{v}_{G}\right)_{T} \\
& =\left(\boldsymbol{R}_{F G T G}\right)_{T} \boldsymbol{M}_{T K}\left(\boldsymbol{v}_{G}\right)_{K}
\end{aligned}
$$

where $\boldsymbol{M}_{T K}$ is the transformation matrix from frame $\{K\}$ to frame $\{T\}$, detailed definition of frame $\{K\}$ and calculation for $\boldsymbol{M}_{T K}$ can be found in Appendix-3, $\chi_{k}$ and $\gamma_{k}$ appeared in $\boldsymbol{M}_{T K}$ are aircraft's flight path azimuth angle and flight path inclination angle.

\section{Trajectory Error Regulator}

The proposed guidance control is an error dynamic based method that calculates the second order error to guide the aircraft to capture and track a desired trajectory path. Trajectory errors and corresponding derivatives $\Delta y_{T}, \Delta z_{T}, \Delta \dot{y}_{T}, \Delta \dot{z}_{T}$ are derived in the previous section and are regarded as inputs to obtain desired aircraft's lateral and vertical accelerations using PD control method as follows:

$$
\begin{aligned}
\Delta \ddot{y}_{T, \text { des }} & =-K_{P Y} \cdot \Delta y_{T}-K_{D Y} \cdot \Delta \dot{y}_{T} \\
\Delta \ddot{z}_{T, \text { des }} & =-K_{P Z} \cdot \Delta z_{T}-K_{D Z} \cdot \Delta \dot{z}_{T}
\end{aligned}
$$

Gains for the above PD controllers are designed in the following fashion, where $\omega_{n y}, \omega_{n z}, \zeta_{y}, \zeta_{z}$ are bandwidth and damping ratio of lateral and vertical trajectory error regulators, respectively. Note that it is not necessary to control the trajectory error in the $x_{T}$ direction explicitly, whereas auto-throttle to maintain the calibrated airspeed is utilized in this paper.

$$
\begin{gathered}
K_{P Y}=\omega_{n y}^{2}, K_{D Y}=2 \zeta_{y} \omega_{n y} \\
K_{P Z}=\omega_{n z}^{2}, K_{D Z}=2 \zeta_{z} \omega_{n z}
\end{gathered}
$$

\section{Outer Loop Guidance Laws}

Based on the principles of INDI [16,19], the dynamic equation of trajectory error under frame $\{T\}$ is established subsequently. The acceleration command generated by the trajectory error regulator is used to obtain the control command required by the inner loop through matrix inverse operation and coordinate conversion.

\section{A. Dynamic Equation of Trajectory Error}

The differential acceleration of $\boldsymbol{s}_{F G}$ under frame $\{T\}$ can be obtained by taking time derivative of Eq. (5) on both sides. The aircraft's acceleration under $\{T\}$ can be more readily measured under its body fixed frame $\{B\}$ (with its details presented in Appendix-4). 


$$
\begin{aligned}
& \left(\boldsymbol{a}_{F T}\right)_{T}=\left[\begin{array}{lll}
\Delta \ddot{x}_{T} & \Delta \ddot{y}_{T} & \Delta \ddot{z}_{T}
\end{array}\right]^{T} \\
& =\left(\boldsymbol{R}_{F G T G}\right)_{T}\left(\boldsymbol{a}_{G}\right)_{T} \\
& =\left(\boldsymbol{R}_{F G T G}\right)_{T}\left[\boldsymbol{M}_{T B}\left(\boldsymbol{a}_{G}\right)_{B}\right] \\
& =\left(\boldsymbol{R}_{F G T G}\right)_{T}\left[\boldsymbol{M}_{T B}\left(\boldsymbol{N}_{G}\right)_{B} \cdot g+\boldsymbol{M}_{T N}(\boldsymbol{g})_{N}\right]
\end{aligned}
$$

where $\boldsymbol{M}_{T B}$ is the transformation matrix from body frame $\{B\}$ to frame $\{T\}$ and can be calculated via $\boldsymbol{M}_{T B}=\boldsymbol{M}_{T \bar{B}} \boldsymbol{M}_{\bar{B} N}$ using the results presented in Appendix-4; $\boldsymbol{M}_{T N}$ is the transformation matrix form navigation frame $\{N\}$ to frame $\{T\} ;\left(N_{G}\right)_{B}$ is aircraft's load factor expressed in frame $\{B\} ;(\boldsymbol{g})_{N}$ is gravity acceleration expressed in frame $\{N\}$, whereas $g$ is the scalar value of the gravity acceleration.

Noteworthily, the above equation connects the trajectory error acceleration with aircraft's bank angle roll angle $\phi$ and normal load factor $\left(N_{G z}\right)_{B}$, which allows the usage of $\phi$ and $\left(\boldsymbol{N}_{G z}\right)_{B}$ as control commends to eliminate trajectory error.

Since trajectory error in the $x_{T}$ direction is not controlled, Eq. (10) is dimensional-reduced onto $y, z$ directions separately or onto both of them to form two novel INDI based guidance methods: lateral and vertical decoupled guidance law, and lateral and vertical coupled guidance law.

\section{Method 1: Lateral and Vertical Decoupled Guidance Law}

Consider the second order lateral error acceleration under frame $\{T\}$, namely $\Delta \ddot{y}_{T}$, by extracting the y component from Eq. (10):

$$
\begin{aligned}
\Delta \ddot{y}_{T} & =\boldsymbol{S}_{y}\left(\boldsymbol{R}_{F G T G}\right)_{T}\left[\boldsymbol{M}_{T B}\left(\boldsymbol{N}_{G}\right)_{B} \cdot g+\boldsymbol{M}_{T N}(\boldsymbol{g})_{N}\right] \\
& =\boldsymbol{S}_{y} \boldsymbol{M}_{T B}\left(\boldsymbol{N}_{G}\right)_{B} \cdot g+\boldsymbol{S}_{y} \boldsymbol{M}_{T N}(\boldsymbol{g})_{N} \\
& =\boldsymbol{S}_{y} \boldsymbol{M}_{T B}\left(\boldsymbol{N}_{G}\right)_{B} \cdot g+0, \Delta \psi_{T} \triangleq \psi-\chi_{T} \\
& \approx\left(N_{G x}\right)_{B} \cdot g \cdot s \Delta \psi_{T} c \theta+\left(N_{G z}\right)_{B} \cdot g\left(s \Delta \psi_{T} s \theta c \phi-c \Delta \psi_{T} s \phi\right)
\end{aligned}
$$

where $\boldsymbol{S}_{y}=[0,1,0]$ is row selection vector, and $\left(\boldsymbol{N}_{G}\right)_{B}=\left[\left(N_{G x}\right)_{B}, 0,\left(N_{G z}\right)_{B}\right]^{T}$ is aircraft's load factor in body frame $\{B\},\left(N_{G x}\right)_{B}$ and $\left(N_{G Z}\right)_{B}$ are $x$ and $Z$ components. The $y$ component is very small and can be ignored.

Equation (11) indicates that the lateral error acceleration under frame $\{T\}$ is a nonlinear/non-affine function of roll angle $\phi$ which can be regarded as a virtual surface input to control lateral error. Bear in mind the principle of INDI method can we get $\phi$ command:

$$
\begin{gathered}
\left(\frac{\partial \Delta \ddot{y}_{T}}{\partial \phi}\right)^{-1}=\left(N_{G z}\right)_{B} \cdot g\left(s \Delta \psi_{T} s \theta s \phi+c \Delta \psi_{T} c \phi\right) \\
\phi_{c m d}=\phi+\left(\frac{\partial \Delta \ddot{y}_{T}}{\partial \phi}\right)^{-1}\left(\Delta \ddot{y}_{T, d e s}-\Delta \ddot{y}_{T}\right) \\
=\phi-\frac{\Delta \ddot{y}_{T, d e s}-\Delta \ddot{y}_{T}}{\left(N_{G z}\right)_{B} \cdot g\left(s \Delta \psi_{T} s \theta s \phi+c \Delta \psi_{T} c \phi\right)}
\end{gathered}
$$


Next, consider the vertical error acceleration under frame $\{T\}$. Similar to the previous derivation, the $z$ component $\Delta \ddot{z}_{T}$ can be extracted from Eq. (10) using a selection vector $\boldsymbol{S}_{Z}=[0,0,1]$ :

$$
\begin{aligned}
\Delta \ddot{z}_{T} & =\boldsymbol{S}_{Z}\left(\boldsymbol{R}_{F G T G}\right)_{T}\left[\boldsymbol{M}_{T B}\left(\boldsymbol{N}_{G}\right)_{B} \cdot g+\boldsymbol{M}_{T N}(\boldsymbol{g})_{N}\right] \\
& =\boldsymbol{S}_{Z} \boldsymbol{M}_{T B}\left(\boldsymbol{N}_{G}\right)_{B} \cdot g+\boldsymbol{S}_{Z} \boldsymbol{M}_{T N}(\boldsymbol{g})_{N} \\
& \approx\left(N_{G x}\right)_{B} \cdot g \cdot\left(s \gamma_{T} c \theta c \Delta \psi_{T}-c \gamma_{T} s \theta\right) \\
& +\left(N_{G Z}\right)_{B} \cdot g\left[s \gamma_{T} s \Delta \psi_{T} s \phi+\left(s \gamma_{T} c \Delta \psi_{T} s \theta+c \gamma_{T} c \theta\right) c \phi\right]+g c \gamma_{T}
\end{aligned}
$$

The above equation is an affine function of aircraft's normal load factor $\left(\boldsymbol{N}_{G Z}\right)_{B}$. Subsequently, the normal load factor command can be obtained by solving Eqn. (14):

$$
\left(N_{G z, c m d}\right)_{B}=\frac{\frac{\Delta \ddot{z}_{T, d e s}}{g \cdot c \gamma_{T}}-\left(N_{G x}\right)_{B}\left(\tan \gamma_{T} c \theta c \Delta \psi_{T}-s \theta\right)-1}{\tan \gamma_{T} s \Delta \psi_{T} s \phi+\left(\tan \gamma_{T} s \theta c \Delta \psi_{T}+c \theta\right) c \phi}
$$

\section{Method 2: Lateral and Vertical Coupled Guidance Law}

Taken the fact that the longitudinal and the lateral channels of an aircraft are coupled, which are decoupled to establish the previous guidance control method, into account, a second method is proposed and derived here. Consider the second order error acceleration in $y$ and $z$ directions simultaneously in the frame $\{T\}$ :

$$
\begin{aligned}
& {\left[\begin{array}{c}
\Delta \ddot{y}_{T} \\
\Delta \ddot{z}_{T}
\end{array}\right]=\boldsymbol{S}_{y z}\left(\boldsymbol{R}_{F G T G}\right)_{T}\left[\boldsymbol{M}_{T B}\left(\boldsymbol{N}_{G}\right)_{B} \cdot g+\boldsymbol{M}_{T N}(\boldsymbol{g})_{N}\right]} \\
& =\boldsymbol{S}_{y z} \boldsymbol{M}_{T B}\left(\boldsymbol{N}_{G}\right)_{B} \cdot \boldsymbol{g}+\boldsymbol{S}_{y z} \boldsymbol{M}_{T N}(\boldsymbol{g})_{N}
\end{aligned}
$$

where the selection matrix $\boldsymbol{S}_{y z}=[0,1,0 ; 0,0,1]$ picks both the $y$ and $z$ channels out. Similar to the previous decoupled method, in order to obtain roll angle command and normal load factor command, partial derivatives of the above equation are taken with respect to $\phi$ and $\left(N_{G Z}\right)_{B}$. Note that $\boldsymbol{M}_{T N}$ is independent from $\phi$ or $\left(N_{G z}\right)_{B}$ (see Appendix-4 for more details), resulting in zero for related partial derivative terms.

$$
\begin{aligned}
& \frac{\partial\left[\begin{array}{ll}
\Delta \ddot{y}_{T} & \Delta \ddot{z}_{T}
\end{array}\right]^{T}}{\partial\left[\begin{array}{ll}
\phi & \left(N_{G z}\right)_{B}
\end{array}\right]}=g \frac{\partial \boldsymbol{S}_{y z} \boldsymbol{M}_{T B}\left(\boldsymbol{N}_{G}\right)_{B}}{\partial\left[\begin{array}{ll}
\phi & \left(N_{G z}\right)_{B}
\end{array}\right]} \\
& =g \frac{\partial \boldsymbol{S}_{y z} \boldsymbol{M}_{T \bar{B}} M_{\bar{B} B}\left(\boldsymbol{N}_{G}\right)_{B}}{\partial\left[\phi\left(N_{G z}\right)_{B}\right]} \\
& =g \boldsymbol{S}_{y z} \boldsymbol{M}_{T \bar{B}} \frac{\partial M_{\bar{B} B}\left(\boldsymbol{N}_{G}\right)_{B}}{\partial\left[\begin{array}{ll}
\phi & \left(N_{G Z}\right)_{B}
\end{array}\right]} \\
& =g\left[\begin{array}{ll}
\boldsymbol{M}_{T \bar{B}}(2,2) & \boldsymbol{M}_{T \bar{B}}(2,3) \\
\boldsymbol{M}_{T \bar{B}}(3,2) & \boldsymbol{M}_{T \bar{B}}(3,3)
\end{array}\right]\left[\begin{array}{cc}
-\left(N_{G Z}\right)_{B} c \phi & -s \phi \\
-\left(N_{G Z}\right)_{B} s \phi & c \phi
\end{array}\right]
\end{aligned}
$$

The incremental commands of roll angle and normal load factor can be obtained using again the INDI principle: 


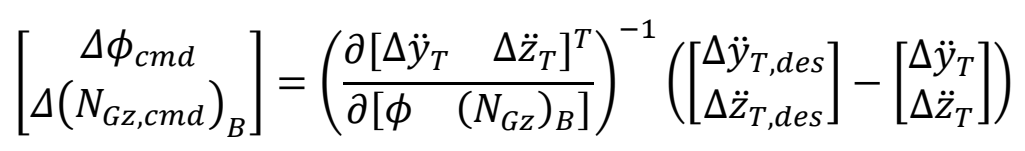

Finally, the INDI based coupled guidance commands are:

$$
\left[\begin{array}{c}
\phi_{c m d} \\
\left(N_{G z, c m d}\right)_{B}
\end{array}\right]=\left[\begin{array}{c}
\phi \\
\left(N_{G z}\right)_{B}
\end{array}\right]+\left[\begin{array}{c}
\Delta \phi_{c m d} \\
\Delta\left(N_{G z, c m d}\right)_{B}
\end{array}\right]
$$

\section{B. Stability-Axis Roll Rate Command}

The motion equation of an aircraft [18] is listed below with its detailed calculation progress presented in the Appendix-6.

$$
\begin{gathered}
P_{s}=P c \alpha+R s \alpha \\
\dot{\phi}=P+(Q s \phi+R c \phi) \tan \theta
\end{gathered}
$$

where $P_{S}$ is roll rate around an aircraft's stability axis; $P, Q, R$ are conventional roll rate, pitch rate and yaw rate, respectively; $\alpha$ is the angle of attack (AoA).

Given a desired roll rate command $\dot{\phi}_{\text {des }}$ and measuring other critical variables, the roll rate command around an aircraft's $\mathrm{x}$ stability axis is acquired by solving Eqs. (20-21):

$$
P_{s, c m d}=c \alpha\left[\dot{\phi}_{d e s}-Q s \phi \tan \theta+R(\tan \alpha-c \phi \tan \theta)\right]
$$

The desired roll rate command is given by the roll incremental times a gain $K_{\phi}$ :

$$
\dot{\phi}_{\text {des }}=K_{\phi}\left(\phi_{c m d}-\phi\right)
$$

where $\phi_{c m d}$ is obtained via Eq. 12 for uncoupled method and via Eq. 19 for coupled method.

\section{Simulation and Results}

To straightforwardly demonstrate the functionality and validate the performance of the proposed guidance law, a six DoF non-linear aircraft simulation platform, along with necessary sensor and conventional actuator modules, is established in Matlab Simulink environment. Three common flight modes are tested and analyzed. Specifically, the LOC/GLS mode for an aircraft to track a glideslope, the LOC/ALT mode for an aircraft to track a localizer course and altitude, and the PHI/ALT mode for an aircraft to perform roll maneuver while holding altitude. Note that only the outer loop that generates guidance commends in the form of 
$\left(N_{G z, c m d}\right)_{B}$ and/or $P_{S, c m d}$ is considered here in this work, while the inner loops which are assumed to have satisfactorily good control performance fall out of this paper's main focus. Note that for all of the function modes, the tunning required parameters are only those needed for the desired lateral and vertical acceleration calculations using PD control in Eqs. (8-9) and values assigned in the following simulation examples were $\omega_{n y}=0.15, \zeta_{y}=0.8, \omega_{n z}=0.15, \zeta_{z}=0.8$.

\section{A. LOC/GLS Mode}

A straight line with trajectory inclination angle $\gamma_{T}=-5^{\circ}$ and trajectory azimuth angle $\chi_{T}=0^{\circ}$ is given for an aircraft to track the glideslope as an application example in LOC/GLS mode. The aircraft flies initially at the right side of the desired trajectory at distances of $30 \mathrm{~m}$ and $300 \mathrm{~m}$, respectively. The left side situation is not simulated due to symmetricity. Two proposed guidance control methods, namely the uncoupled and coupled guidance control laws, are applied to function in this LOC/GLS mode. Figures 3 and 5 plot out curves of certain critical variables against time, including altitude of the aircraft, lateral and vertical distance errors, Mach number, load factor, and roll angle. Desired trajectories along with actual trajectories of an aircraft guided by the proposed decoupled and coupled methods of the two situations are presented in Figs. 4 and 6, respectively.
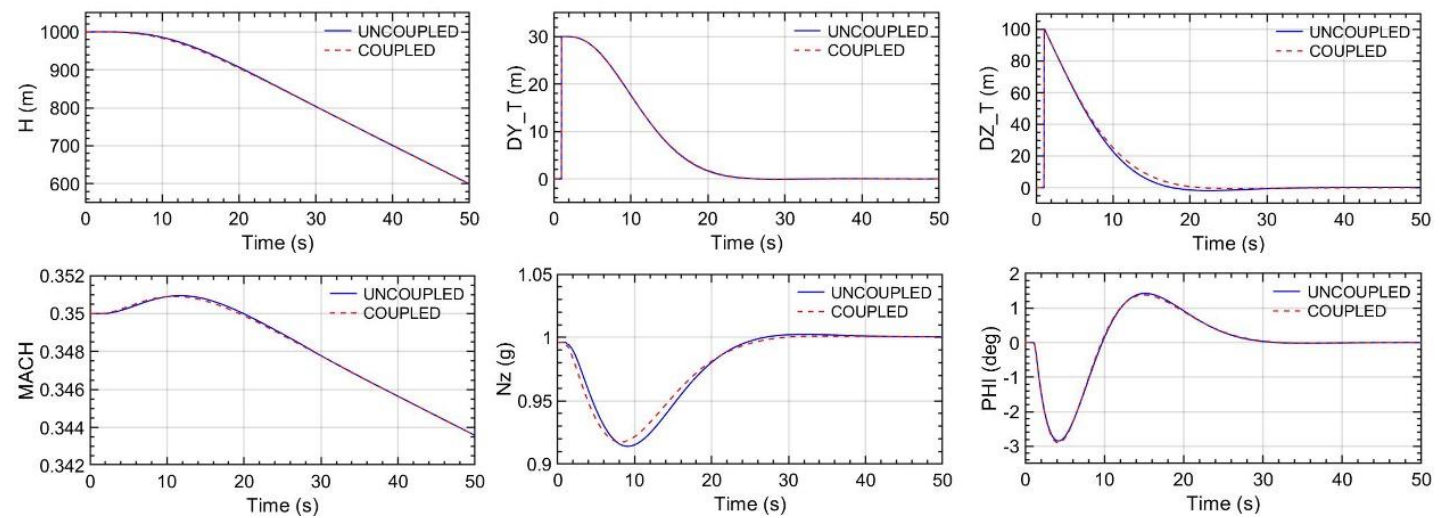

Fig. 3 Critical variables of LOC/GLS mode at initial position of $30 \mathrm{~m}$ to the right and $100 \mathrm{~m}$ below the reference trajectory.

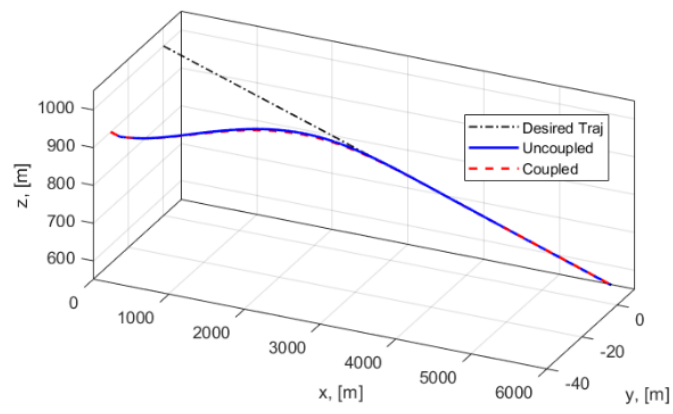


Fig. 4 Trajectories of LOC/GLS mode at initial position: $30 \mathrm{~m}$ to the right and $100 \mathrm{~m}$ below the reference trajectory.
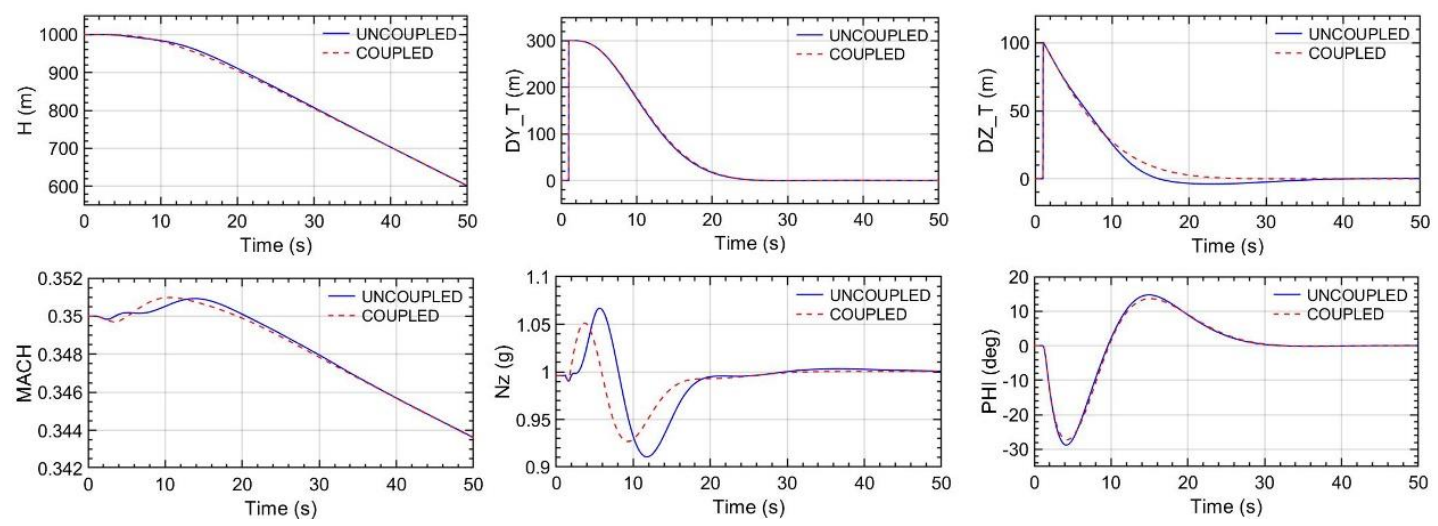

Fig. 5 Critical variables of LOC/GLS mode at initial position of $300 \mathrm{~m}$ to the right and $100 \mathrm{~m}$ below the reference trajectory.

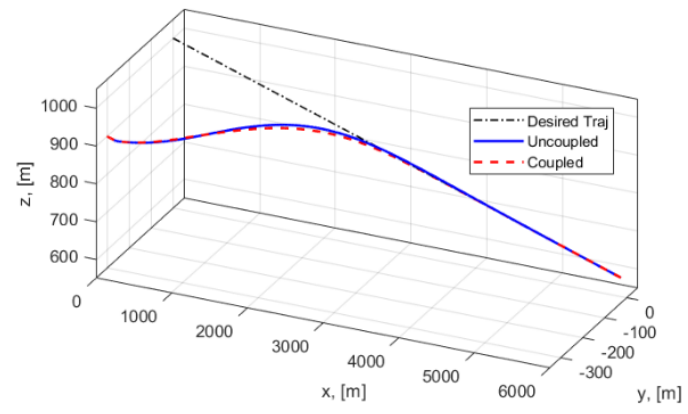

Fig. 6 Trajectories of LOC/GLS mode at initial position of $300 \mathrm{~m}$ to the right and $100 \mathrm{~m}$ below the reference trajectory.

As the general specification [20] requires a specified accuracy of $\pm 10 \mathrm{~m}$ shall be achieved within 30 seconds, the above simulation results demonstrate that the aircraft controlled under either of the proposed two guidance methods can intercept and track the reference straight-line trajectory quickly and nicely. The lateral distance errors for the $30 \mathrm{~m}$ and $300 \mathrm{~m}$ cases vanish at around 24 seconds and are achieved by rolling towards left side since the aircraft initially flies at the right side of the desired trajectory. For the vertical error, two methods performed roughly the same when the initial lateral error is small (i.e. $30 \mathrm{~m}$ ). The uncoupled method shows a little overshot, meaning the aircraft flies under the desired trajectory slightly for a short time for the non-vicinity case (large initial vertical error, i.e. $300 \mathrm{~m}$, case), and it takes approximately 30 seconds to diminish the error. The couped method, on the other hand, illustrates a better performance and reaches within the specified accuracy faster. Lastly, the aircraft controlled by the coupled method responds faster with smaller load factor variations, resulting from the fact a better optimized control 
allocation is obtained because the lateral and vertical channels are controlled conjugately, and hence various actuators cooperate better than the uncoupled method.

\section{B. LOC/ALT Mode}

A straight line with trajectory inclination angle $\gamma_{T}=0^{\circ}$ and trajectory azimuth angle $\chi_{T}=0^{\circ}$ is given for an aircraft to track and maintain at a certain altitude. Similar to the previous LOC/GLS mode, the aircraft flies initially at the right side of the desired trajectory at distances of $30 \mathrm{~m}$ and $300 \mathrm{~m}$ and at an initial height of $1000 \mathrm{~m}$. To study and analyze the guidance control performance, two desired altitudes are set as the target altitudes, requiring the aircraft to perform a pull or push maneuver. Small lateral and vertical distance error situations, specifically $30 \mathrm{~m}$ below and above the desired altitudes, are plotted in Figs. 7-10. And figures 11-14 compare the nonvicinity near the desired trajectory (larger distance error) situations, providing results from both coupled and decoupled control methods. Desired trajectories with aircraft's actual flight trajectories under guidance control of both decoupled and coupled methods are shown in Figs. 8,10,12,14 for the four simulation cases respectively.
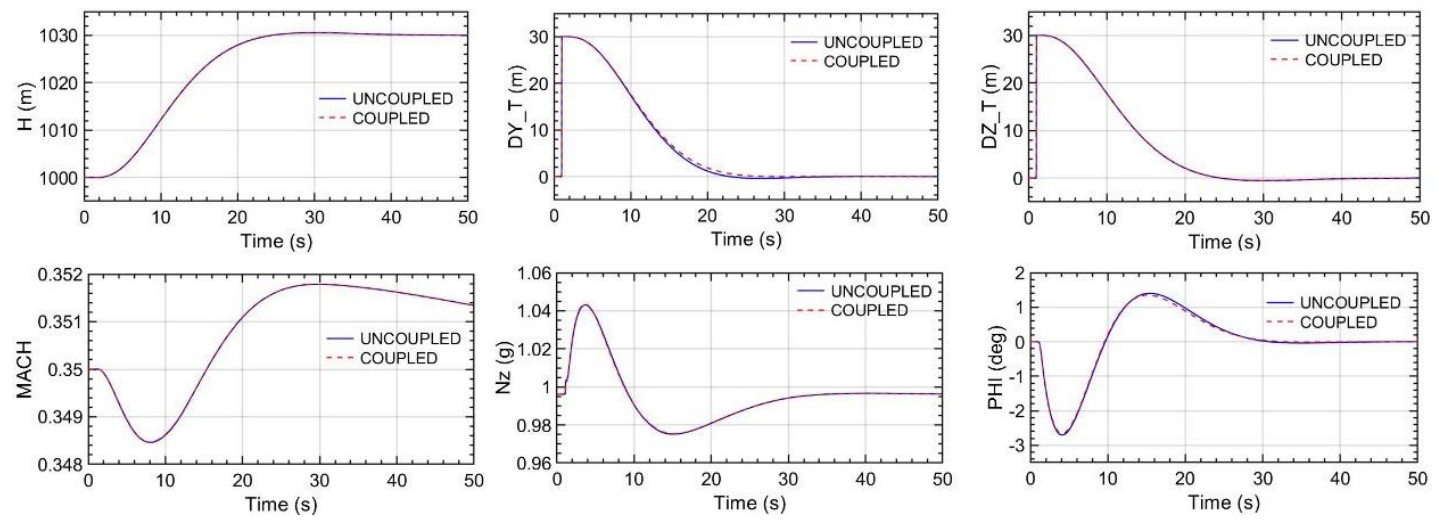

Fig. 7 Critical variables of LOC/ALT mode at initial position of $30 \mathrm{~m}$ to the right and $30 \mathrm{~m}$ below the reference trajectory.

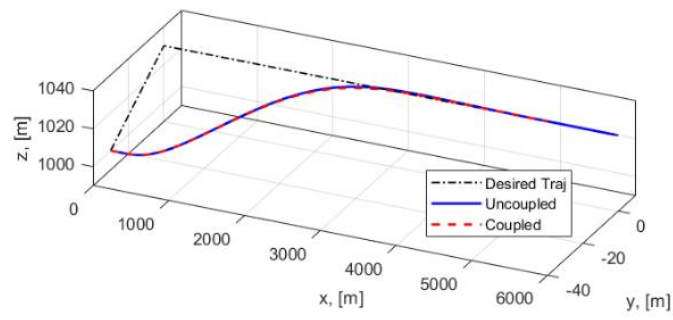

Fig. 8 Trajectories of LOC/ALT mode at initial position of $30 \mathrm{~m}$ to the right and $30 \mathrm{~m}$ below the reference trajectory. 

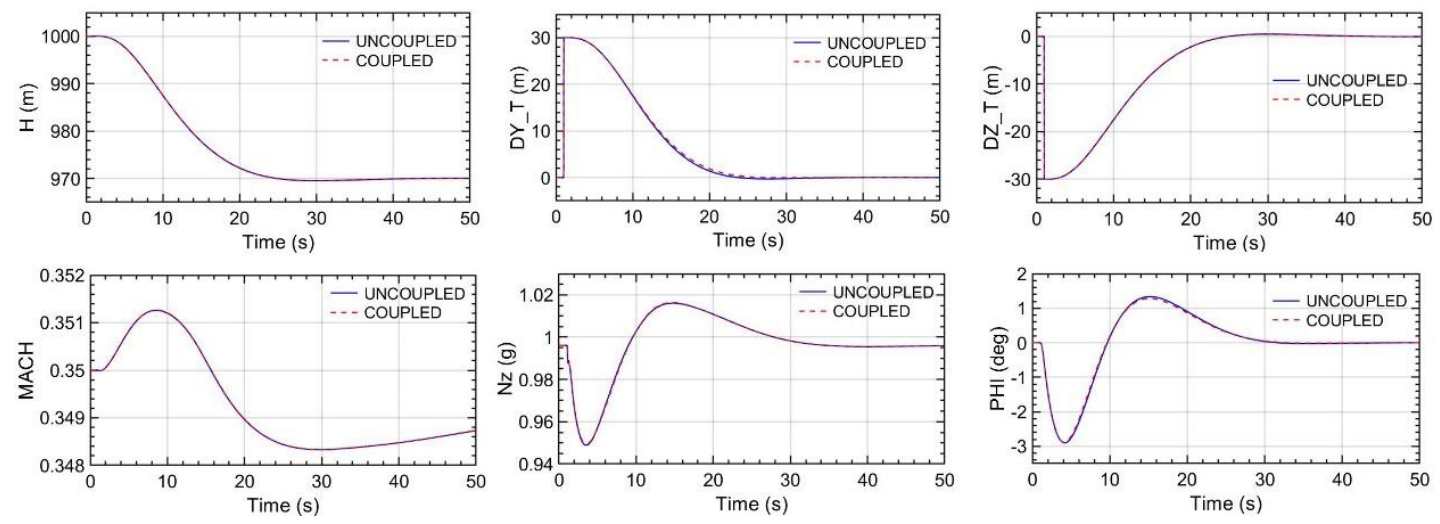

Fig. 9 Critical variables of LOC/ALT mode at initial position of $30 \mathrm{~m}$ to the right and $30 \mathrm{~m}$ above the reference trajectory.

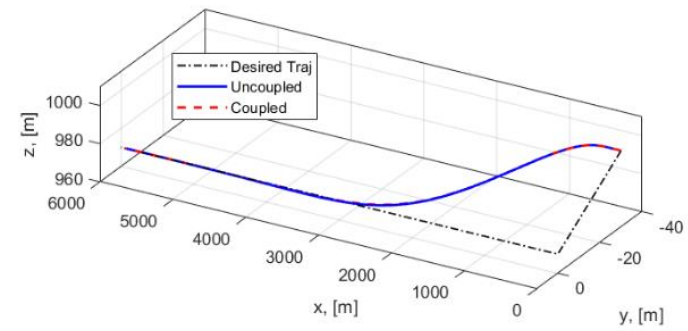

Fig. 10 Trajectories of LOC/ALT mode at initial position of $30 \mathrm{~m}$ to the right and $30 \mathrm{~m}$ above the reference trajectory.

When the aircraft under guidance flies near the desired trajectory, both of the proposed guidance control methods illustrate good performances and satisfy the aforementioned specification, in the pull-stick and the push-stick autopilot situations. The longitudinal errors, for $h_{c m d}=1030 \mathrm{~m}$ and $970 \mathrm{~m}$, are gradually reduced to zero and the desired altitudes are therefore achieved nicely. For lateral errors, the uncoupled method induces a little bit overshot in both cases, meaning the aircraft flies across over the vertical plane determined by the desired trajectory and then flies back. The coupled method, to the contrary, shows no overshot and performs better. Nonetheless, the two proposed methods generally illustrate equivalently good performance in small initial error situations. 

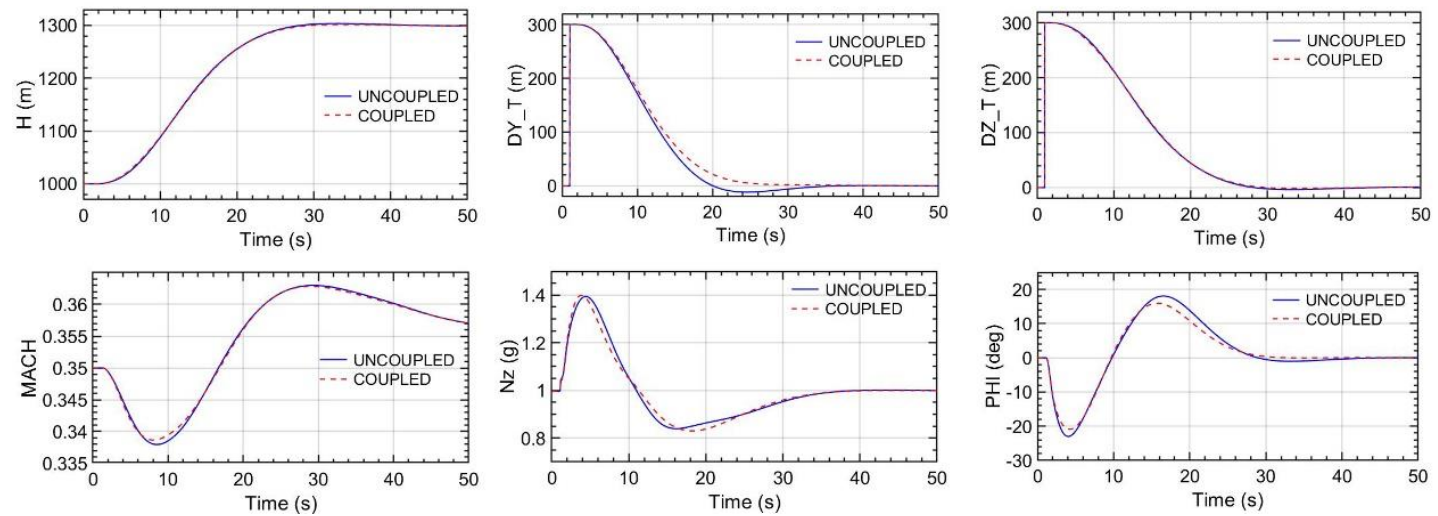

Fig. 11 Critical variables of LOC/ALT mode at initial position of $300 \mathrm{~m}$ to the right and $300 \mathrm{~m}$ below the reference trajectory.

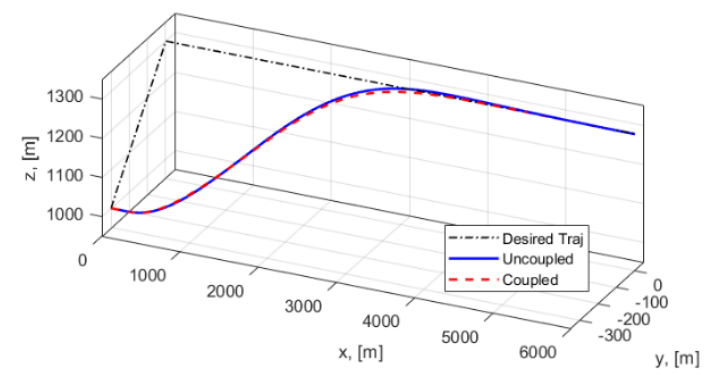

Fig. 12 Trajectories of LOC/ALT mode at initial position of $300 \mathrm{~m}$ to the right and $300 \mathrm{~m}$ below the reference trajectory.
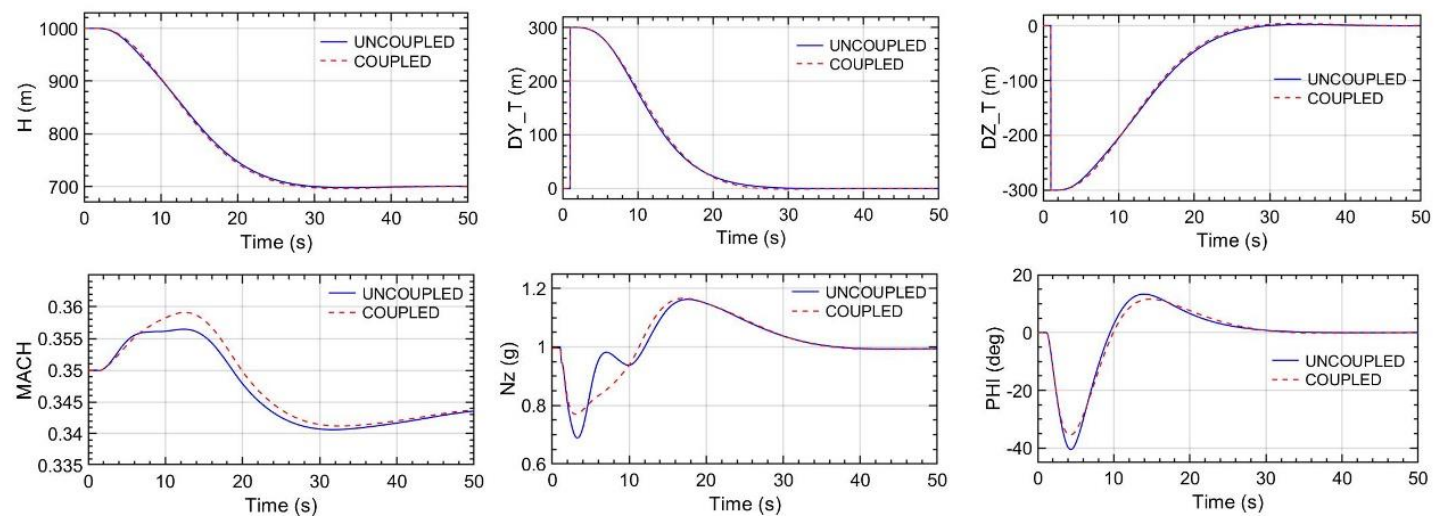

Fig. 13 Critical variables of LOC/ALT mode at initial position of $300 \mathrm{~m}$ to the right and $300 \mathrm{~m}$ above the reference trajectory. 


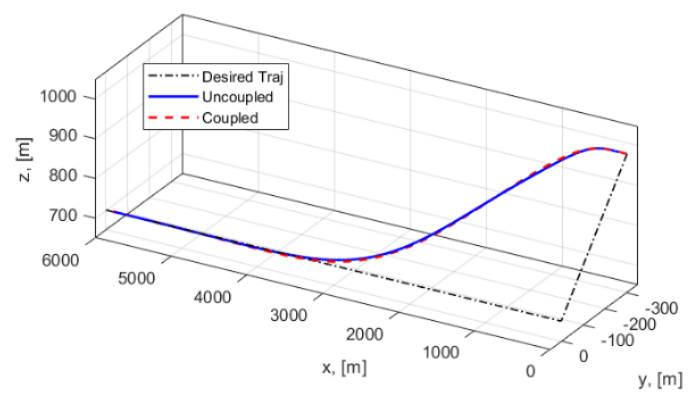

\section{Fig. 14 Trajectories of LOC/ALT mode at initial position of $300 \mathrm{~m}$ to the right and $300 \mathrm{~m}$ above the reference trajectory.}

For the non-vicinity near the desired trajectory case, both guidance control methods fulfill the general specifications when the aircraft initially has large lateral and vertical distance errors. The uncouple method leads the aircraft with some tiny overshoot in the push-down control situation, while no obvious overshoot is observed in the pull-up flight. The reason behind may be derived from the roll angle plot as the aircraft rolls more in the climbing situation when controlled by the uncoupled method and needs to roll back essentially. In the descending situation, both methods show similar roll maneuvers. Noteworthily, the aircraft controlled by the uncoupled method experiences larger load factor variations, specially in the pushdown situation, while one controlled by the coupled method shows gentler and smoother curves.

\section{PHI/ALT Mode}

An aircraft is controlled to track a desired altitude and also to perform lateral maneuvers to track a roll angle command. In this case, the trajectory inclination angle $\gamma_{T}=0^{\circ}$ and let $\Delta \psi=0$. A roll command of $30^{\circ}$ is given at 25 seconds. Note that the lateral trajectory control is ignored since a roll command instead of a desired lateral position is given, and therefore only the uncoupled method is applied here. Four desired altitudes, i.e. 1030m, 970m, 1300m and 700m, are set as altitude commands to evaluate the guidance performance for vicinity and non-vicinity cases. Related variables are scoped in Figs. 15 and 16. As PHI/ALT mode specifies a roll command instead of an explicitly desired trajectory, trajectory results are not plotted out here like the previous modes. 

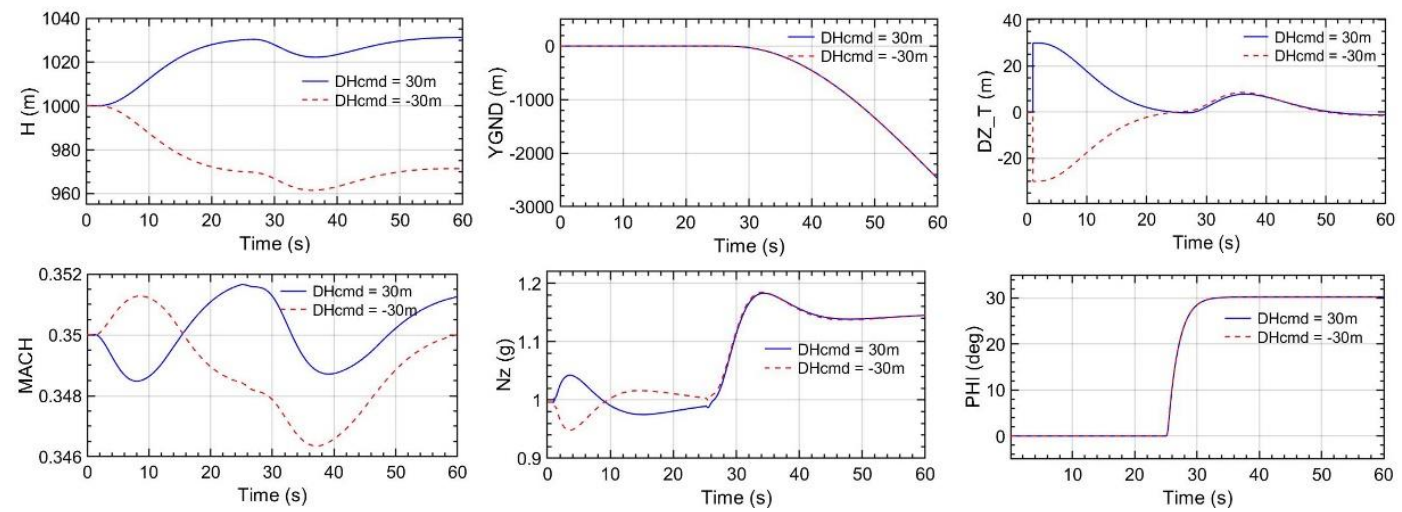

Fig. 15 Critical variables of PHI/ALT mode at initial position of $30 \mathrm{~m}$ above/below the reference trajectory, Maneuver: $30^{\circ}$ roll at 25 sec.
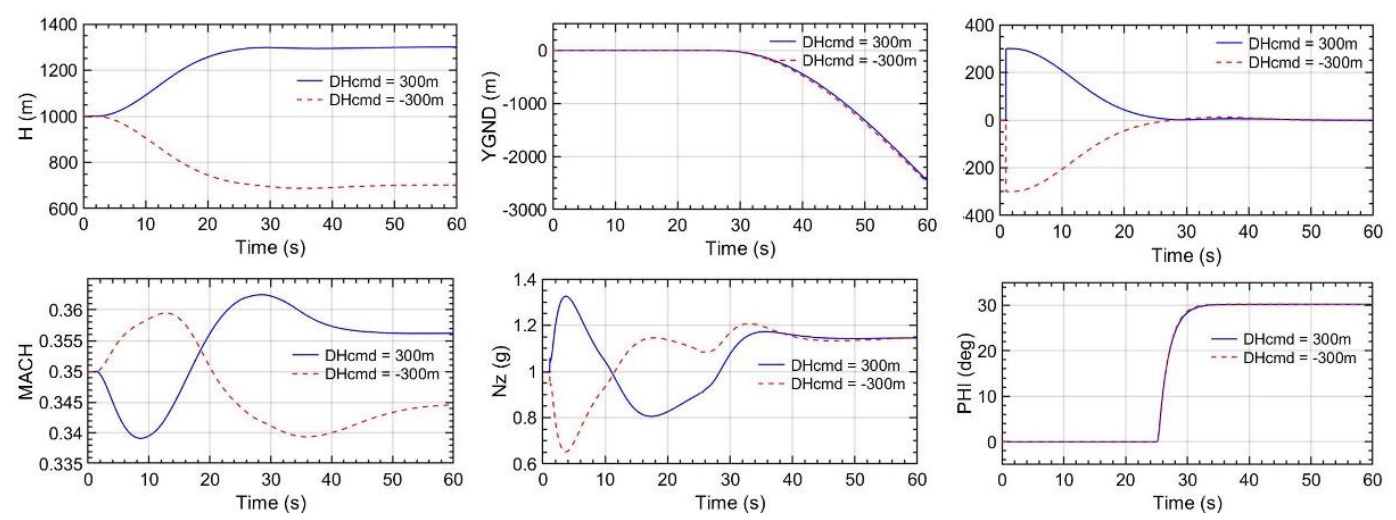

Fig. 16 Critical variables of PHI/ALT mode at initial position of $300 \mathrm{~m}$ above/below the reference trajectory, Maneuver: $30^{\circ}$ roll at $25 \mathrm{sec}$.

For the 30m climbing and descending situations, the vertical errors bump up from zero at the moment when the aircraft starts to roll because the rolling maneuver inevitably induces lift in vertical direction. Still, the aircraft guided by the proposed method takes roughly 25 seconds to reduce the error to null and to achieve the altitude maintenance also. Once the aircraft reaches the "steady" turn state, a constant load factor should be induced, as the third subgraph in Figs. 14 and 15 show. For the $300 \mathrm{~m}$ altitude case, analogous results are obtained, and similar analyzation results can be drawn. Conclusively, the aircraft guided by the proposed method demonstrates satisfactory performance according to the general specifications.

\section{Conclusion}

Current classical guidance law for a fixed wing aircraft, namely a fighter jet, conventionally decouples longitudinal and lateral commands, resulting in poor flight performance in large maneuvers. Even under small maneuvers, the aircraft needs to be close to the reference trajectory. Besides, classical guidance law requires numerous parameter tunning since the physical concept behind is not so clear. Furthermore, necessary lead-lag compensations are needed to improve system stability margin due to the same reason. 
Therefore, aiming at the capturing and tracking of fixed linear reference trajectories, a novel INDI based guidance law for fixed wing aircraft is proposed in this paper. The proposed trajectory guidance method, namely the outer loop, takes conventional or measurable variables from an aircraft and associated sensors as inputs. This outer loop is modular designed and is capable to work with versatile inner loop control methods since it produces conventional longitudinal command $N_{G z, c m d}$ and lateral command $P_{s, c m d}$. Therefore, a novel modular guidance control method is offered to the community to apply onto various platforms and could relieve stress of the tedious control parameter tuning issue.

The proposed guidance methods are mathematically derived in detail, where one decouples the lateral and the longitudinal channels while the second takes the interactions into account. The cumbersome mathematical operations involved in the derivation process aim at reaching a more concise control method and also at providing the community with clearer physical concepts behind this formula. The reason for manipulating transformation matrices is to find a univariate function and to isolate the variable as a virtual input. Based on this, the principle of the well-studied INDI method can be used and a control function between desired or commanded variable and controlled variable is established.

Illustrative application examples including LOC/GLS, LOC/ALT and ALT/PHI modes are simulated upon a 6 DoF nonlinear flight simulation platform to demonstrate the advantages of the proposed methods. The number of tunning parameters necessary for the control design is dramatically reduced. The aircraft under control shows satisfactory flight performance judged by the MIL-F-9490D military specification, even it is not that close to the desired trajectory. Conclusively, the proposed method can achieve precise trajectory tracking and demonstrate satisfactory dynamic characteristics and robustness.

\section{References}

[1] Mahony, R., Kumar, V., \& Corke, P. (2012). Multirotor aerial vehicles: Modeling, estimation, and control of quadrotor. IEEE Robotics and Automation magazine, 19(3), 20-32.

[2] Fresk, E., \& Nikolakopoulos, G. (2013, July). Full quaternion based attitude control for a quadrotor. In 2013 European control conference (ECC) (pp. 38643869). IEEE.

[3] Stephan, J., Pfeifle, O., Notter, S., Pinchetti, F., \& Fichter, W. (2020). Precise Tracking of Extended Three-Dimensional Dubins Paths for Fixed-Wing Aircraft. Journal of Guidance, Control, and Dynamics, 43(12), 2399-2405.

[4] Schatz, S. P., \& Holzapfel, F. (2014, November). Modular trajectory/path following controller using nonlinear error dynamics. In 2014 IEEE International Conference on Aerospace Electronics and Remote Sensing Technology (pp. 157-163). IEEE.

[5] Yamasaki, T., Balakrishnan, S. N., \& Takano, H. (2013). Separate-channel integrated guidance and autopilot for automatic path-following. Journal of Guidance, Control, and Dynamics, 36(1), 25-34.

[6] Kaminer, I., Pascoal, A., Xargay, E., Hovakimyan, N., Cao, C., \& Dobrokhodov, V. 
(2010). Path following for small unmanned aerial vehicles using L1 adaptive augmentation of commercial autopilots. Journal of guidance, control, and dynamics, 33(2), 550-564.

[7] Lawrence, D. A., Frew, E. W., \& Pisano, W. J. (2008). Lyapunov vector fields for autonomous unmanned aircraft flight control. Journal of Guidance, Control, and Dynamics, 31(5), 1220-1229.

[8] Nelson, D. R., Barber, D. B., McLain, T. W., \& Beard, R. W. (2007). Vector field path following for miniature air vehicles. IEEE Transactions on Robotics, 23(3), 519-529.

[9] Medagoda, E. D., \& Gibbens, P. W. (2010). Synthetic-waypoint guidance algorithm for following a desired flight trajectory. Journal of guidance, control, and dynamics, 33(2), 601-606.

[10]Zhang, J. M., Li, Q., Cheng, N., \& Liang, B. (2014). Path-following control for fixed-wing unmanned aerial vehicles based on a virtual target. Proceedings of the Institution of Mechanical Engineers, Part G: Journal of Aerospace Engineering, 228(1), 66-76.

[11]Cho, N., Kim, Y., \& Park, S. (2015). Three-dimensional nonlinear differential geometric path-following guidance law. Journal of Guidance, Control, and Dynamics, 38(12), 2366-2385.

[12] Slotine, J. J. E., \& Li, W. (1991). Applied nonlinear control (Vol. 199, No. 1). Englewood Cliffs, NJ: Prentice hall.

[13]Xie, Z., Xia, Y., \& Fu, M. (2011, September). Robust trajectory-tracking method for UAV using nonlinear dynamic inversion. In 2011 IEEE 5th International Conference on Cybernetics and Intelligent Systems (CIS) (pp. 93-98). IEEE.

[14]Oliveira, T., \& Encarnação, P. (2013). Ground target tracking control system for unmanned aerial vehicles. Journal of Intelligent \& Robotic Systems, 69(1), 373387.

[15]Da Costa, R. R., Chu, Q. P., \& Mulder, J. A. (2003). Reentry flight controller design using nonlinear dynamic inversion. Journal of Spacecraft and Rockets, 40(1), 6471.

[16]Smith, P. (1998, August). A simplified approach to nonlinear dynamic inversion based flight control. In 23rd Atmospheric Flight Mechanics Conference (p. 4461).

[17] Smeur, E. J., Chu, Q., \& de Croon, G. C. (2016). Adaptive incremental nonlinear dynamic inversion for attitude control of micro air vehicles. Journal of Guidance, Control, and Dynamics, 39(3), 450-461.

[18]Zipfel, P. H. (2000). Modeling and simulation of aerospace vehicle dynamics. Aiaa.

[19]Li, M., Li, J., Tang, Y., \& Sun, S. (2021). An Extended INDI Approach and Application to Pitch Rate Control Laws Design of an Aircraft. In AIAA AVIATION 2021 FORUM (p. 3005).

[20] Specification, M. (1975). " Flight Control System-Design, Installation and Test of Piloted Aircraft, General Specification for. MIL-F-9490D, 6 June. 


\section{Appendix}

\section{Navigation Frame $\{\boldsymbol{N}\}$}

The Navigation Frame, denoted as frame $\{N\}$, is used to navigate an aircraft. The frame is established as shown in Fig.17, where the origin is an arbitrary (stationary) point on Earth, the $x$-axis is parallel to the local surface and pointing to geographic north pole, the $z$-axis: orthogonal to the local surface and pointing downwards, and the remaining $y$-axis is determined by right hand rule. Note that under the assumptions mentioned previously, the $z$-axis would simply point downwards into the earth, and the $x$-would point towards North all the time, meaning frame NED and frame $\{N\}$ represent the same orientation.

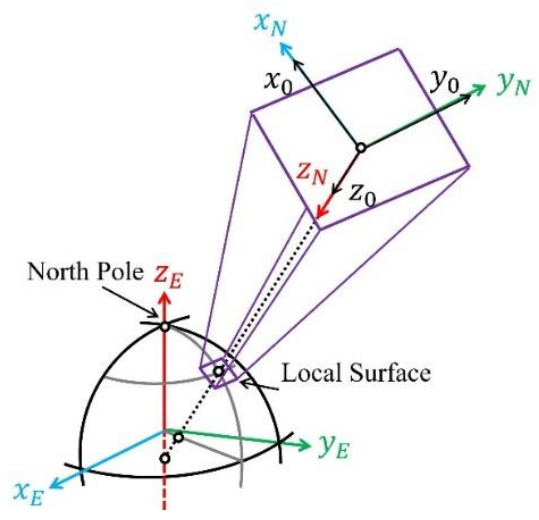

Fig. 17 Navigation Frame $\{\boldsymbol{N}\}$

\section{Reference Trajectory Frame $\{T\}$}

The Reference Trajectory Frame, denoted as frame $\{T\}$, is used to describe the reference trajectory for an aircraft to track. The relation from frame $\{T\}$ to frame $\{N\}$ is shown in Fig.18, and the transformation sequences are: 1) Rotate the frame $\{N\}$ around $z_{N}$ for $\chi_{T}$ and obtain a new frame $\left.\left\{k_{1}, y_{T}, z_{N}\right\} ; 2\right)$ Rotate the new frame $\left\{k_{1}, y_{T}, z_{N}\right\}$ around $y_{T}$ for $\gamma_{T}$ and obtain the frame $\{T\}$. The transformation matrix $\boldsymbol{M}_{T N}$ (and $\boldsymbol{M}_{T O}$ ) is

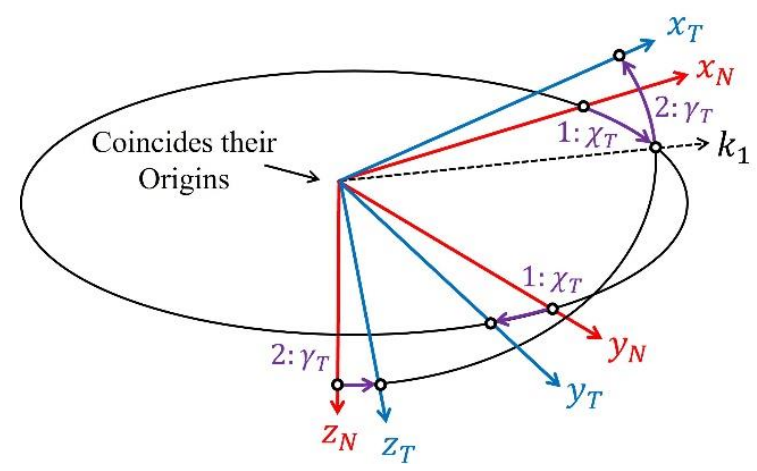

Fig.18 Transformation from frame $\{T\}$ to frame $\{N\}$ 


$$
\begin{aligned}
\boldsymbol{M}_{T N}=\boldsymbol{M}_{T O} & =\left[\begin{array}{ccc}
c \gamma_{T} & 0 & -s \gamma_{T} \\
0 & 1 & 0 \\
s \gamma_{T} & 0 & c \gamma_{T}
\end{array}\right]\left[\begin{array}{ccc}
c \chi_{T} & s \chi_{T} & 0 \\
-s \chi_{T} & c \chi_{T} & 0 \\
0 & 0 & 1
\end{array}\right] \\
& =\left[\begin{array}{ccc}
c \gamma_{T} c \chi_{T} & c \gamma_{T} s \chi_{T} & -s \gamma_{T} \\
-s \chi_{T} & c \chi_{T} & 0 \\
s \gamma_{T} c \chi_{T} & s \gamma_{T} s \chi_{T} & c \gamma_{T}
\end{array}\right]
\end{aligned}
$$

\section{Kinematic Frame $\{K\}$}

The Kinematic Frame, denoted as frame $\{K\}$, is used to describe the velocity vector of an aircraft. The frame is established as shown in Fig.19, where the origin is a reference point on the aircraft, conventionally the Center of Gravity, the X-axis is pointing into the direction of the kinematic velocity of an aircraft, the $y$-axis is pointing to the right perpendicular to the $\mathrm{x}$ - and $\mathrm{z}$-axes the $z$-axis is pointing downwards parallel to the projection of the local normal to the WGS-84 ellipsoid into a plane perpendicular to the $\mathrm{x}$-axis (i.e. to the kinematic velocity). The transformation sequences from NED frame to kinematic frame are: 1) Rotate the frame $\{O\}$ around $z O$ for $\chi_{k}$ and obtain a new frame $\left.\left\{k_{K}, y_{k}, z_{o}\right\} ; 2\right)$ Rotate the new frame $\left\{k_{K}, y_{k}, z_{o}\right\}$ around $y k$ for $\gamma_{k}$ and obtain the frame $\{K\}$.

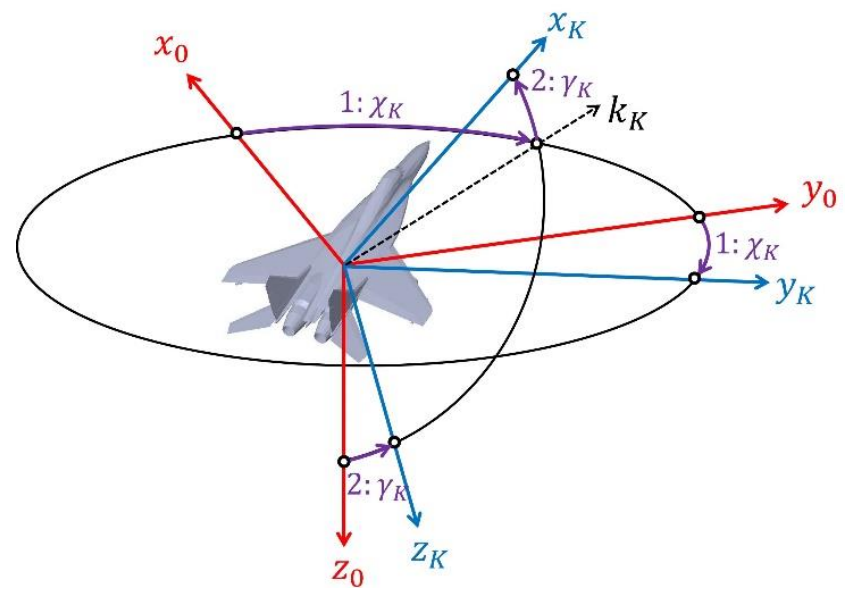

Fig. 19 Transformation from frame $\{O\}$ to frame $\{K\}$

The corresponding transformation matrix $\boldsymbol{M}_{T K}$ is therefore

$\boldsymbol{M}_{T K}=\boldsymbol{M}_{T O} \boldsymbol{M}_{O K}$

$=\boldsymbol{M}_{y}\left(\gamma_{T}\right) \boldsymbol{M}_{z}\left(\chi_{T}\right) \boldsymbol{M}_{z}\left(-\chi_{K}\right) \boldsymbol{M}_{y}\left(-\gamma_{K}\right)$

$=\boldsymbol{M}_{y}\left(\gamma_{T}\right) \boldsymbol{M}_{z}\left(-\Delta \chi_{K}\right) \boldsymbol{M}_{y}\left(-\gamma_{K}\right),\left(\Delta \chi_{K} \triangleq \chi_{K}-\chi_{T}, \Delta \gamma_{K} \triangleq \gamma_{K}-\gamma_{T}\right)$

$=\left[\begin{array}{ccc}c \gamma_{T} & 0 & -s \gamma_{T} \\ 0 & 1 & 0 \\ s \gamma_{T} & 0 & c \gamma_{T}\end{array}\right]\left[\begin{array}{ccc}c \Delta \chi_{K} & -s \Delta \chi_{K} & 0 \\ s \Delta \chi_{K} & c \Delta \chi_{K} & 0 \\ 0 & 0 & 1\end{array}\right]\left[\begin{array}{ccc}c \gamma_{K} & 0 & s \gamma_{K} \\ 0 & 1 & 0 \\ -s \gamma_{K} & 0 & c \gamma_{K}\end{array}\right]$

$=\left[\begin{array}{ccc}c \gamma_{K} c \gamma_{T} c \Delta \chi_{K}+s \gamma_{K} s \gamma_{T} & -s \Delta \chi_{K} c \gamma_{T} & c \gamma_{T} s \gamma_{K} c \Delta \chi_{K}-s \gamma_{T} c \gamma_{K} \\ s \Delta \chi_{K} c \gamma_{K} & c \Delta \chi_{K} & s \Delta \chi_{K} s \gamma_{K} \\ c \gamma_{K} s \gamma_{T} c \Delta \chi_{K}-s \gamma_{K} c \gamma_{T} & -s \Delta \chi_{K} s \gamma_{T} & s \gamma_{T} s \gamma_{K} c \Delta \chi_{K}+c \gamma_{T} c \gamma_{K}\end{array}\right]$

$=\left[\begin{array}{ccc}c \gamma_{K} c \gamma_{T}\left(c \Delta \chi_{K}-1\right)+c \Delta \gamma_{K} & -s \Delta \chi_{K} c \gamma_{T} & c \gamma_{T} s \gamma_{K}\left(c \Delta \chi_{K}-1\right)+s \Delta \gamma_{K} \\ s \Delta \chi_{K} c \gamma_{K} & c \Delta \chi_{K} & s \Delta \chi_{K} s \gamma_{K} \\ c \gamma_{K} s \gamma_{T}\left(c \Delta \chi_{K}-1\right)-s \Delta \gamma_{K} & -s \Delta \chi_{K} s \gamma_{T} & s \gamma_{T} s \gamma_{K}\left(c \Delta \chi_{K}-1\right)+c \Delta \gamma_{K}\end{array}\right]$ 


\section{Body Frame $\{B\}$}

The Body Frame, denoted as frame $\{B\}$, is conventionally used to describe and analyze forces and moments acting on an aircraft. The frame is established where the origin is a reference point on the aircraft, conventionally the Center of Gravity, the $x$ axis is pointing towards the aircraft' head in the symmetry plane, the $y$-axis: pointing towards the starboard side of the aircraft in the wing plane, and the $z$-axis: pointing downwards in the symmetry plane and orthogonal to $x B$ and $y B$ axes. Since the wellknown frame $\{O\}$ and frame $\{B\}$ are established, the transformation in-between and involved angles are incidentally derived here for readers' convenience. As Fig.20 shows, the transformation sequences are: 1) Rotate the frame $\{O\}$ around $z O$ for $\psi$ (yaw angle) and obtain a new frame $\left\{k_{1}, k_{2}, z_{O}\right\} ; 2$ ) Rotate the new frame $\left\{k_{1}, k_{2}, z_{O}\right\}$ around $k 2$ for $\theta$ (pitch angle) and obtain a new frame $\left.\left\{x_{B}, k_{2}, k_{3}\right\} ; 3\right)$ Rotate the new frame $\left\{x_{B}, k_{2}, k_{3}\right\}$ around $x B$ for $\phi$ (roll angle) and obtain the frame $\{B\}$.

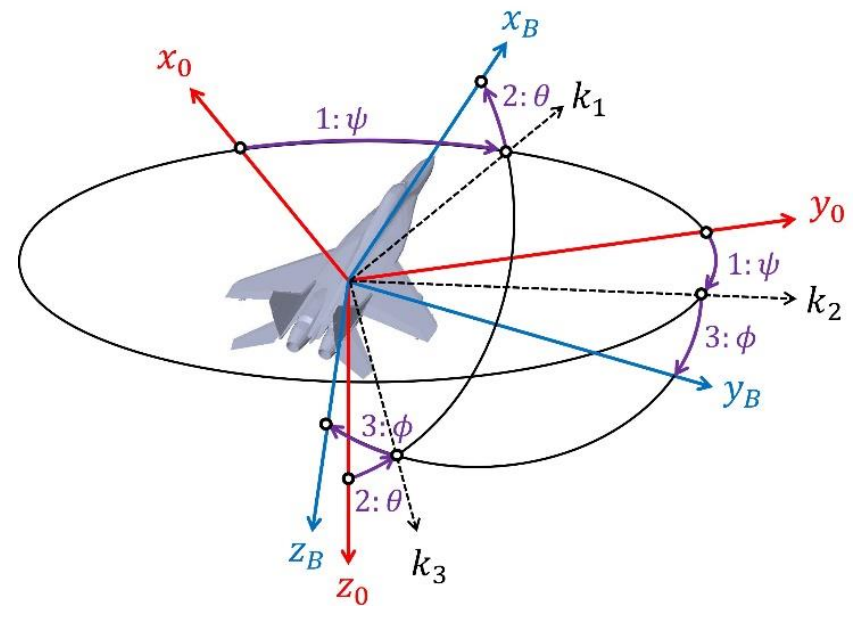

Fig. 20 Transformation from frame $\{O\}$ to frame $\{B\}$

The corresponding transformation matrix $\boldsymbol{M}_{B O}$ (and $\boldsymbol{M}_{B N}$ ) is therefore

$$
\begin{aligned}
& \boldsymbol{M}_{B O}=\boldsymbol{M}_{B N} \\
& =\left[\begin{array}{ccc}
1 & 0 & 0 \\
0 & c \phi & s \phi \\
0 & -s \phi & c \phi
\end{array}\right]\left[\begin{array}{ccc}
c \theta & 0 & -s \theta \\
0 & 1 & 0 \\
s \theta & 0 & c \theta
\end{array}\right]\left[\begin{array}{ccc}
c \psi & s \psi & 0 \\
-s \psi & c \psi & 0 \\
0 & 0 & 1
\end{array}\right] \\
& =\left[\begin{array}{ccc}
c \psi c \theta & s \psi c \theta & -s \theta \\
c \psi s \theta s \phi-s \psi c \phi & c \psi c \phi+s \psi s \theta s \phi & c \theta s \phi \\
c \psi s \theta c \phi+s \psi s \phi & -c \psi s \phi+s \psi s \theta c \phi & c \theta c \phi
\end{array}\right]
\end{aligned}
$$

\section{Intermediate Body Frame $\{\bar{B}\}$}

The Intermediate Body Frame, denoted as frame $\{\bar{B}\}$, is deliberately used in this paper to mathematically simplify for some transformation derivations. The frame is established as shown in Fig.21, where the origin is the same point for frame $\{B\}$. The transformation sequences frame $\{O\}$ and frame $\{\bar{B}\}$ are: 1) Rotate the frame $\{O\}$ around $z_{O}$ for $\psi$ (yaw angle) and obtain a new frame $\left\{k_{1}, y_{\bar{B}}, z_{O}\right\} ; 2$ ) Rotate the new frame $\left\{k_{1}, y_{\bar{B}}, z_{O}\right\}$ around $y_{\bar{B}}$ for $\theta$ (pitch angle) and obtain a new frame $\{\bar{B}\}$. 
The transformation matrix $\boldsymbol{M}_{B \bar{B}}$ from $\{\bar{B}\}$ to $\{B\}$ is straightforward:

$$
\boldsymbol{M}_{B \bar{B}}=\left[\begin{array}{ccc}
1 & 0 & 0 \\
0 & c \phi & s \phi \\
0 & -s \phi & c \phi
\end{array}\right]
$$

A useful transformation from frame $\{N\}$ to frame $\{\bar{B}\}$ can be acquired by manipulating the transformation sequences:

$$
\begin{aligned}
& \boldsymbol{M}_{\bar{B} N}=\boldsymbol{M}_{\bar{B} B} \boldsymbol{M}_{B N}=\boldsymbol{M}_{B \bar{B}} \boldsymbol{M}_{B O} \\
& =\left[\begin{array}{ccc}
1 & 0 & 0 \\
0 & c \phi & s \phi \\
0 & -s \phi & c \phi
\end{array}\right]\left[\begin{array}{ccc}
c \psi c \theta & s \psi c \theta & -s \theta \\
c \psi s \theta s \phi-s \psi c \phi & c \psi c \phi+s \psi s \theta s \phi & c \theta s \phi \\
c \psi s \theta c \phi+s \psi s \phi & -c \psi s \phi+s \psi s \theta c \phi & c \theta c \phi
\end{array}\right] \\
& =\left[\begin{array}{ccc}
c \psi c \theta & s \psi c \theta & -s \theta \\
-s \psi & c \psi & 0 \\
c \psi s \theta & s \psi s \theta & c \theta
\end{array}\right]
\end{aligned}
$$

Another useful transformation from frame $\{\bar{B}\}$ to frame $\{T\}$ is obtained by definitions of transformation matrices $\boldsymbol{M}_{T O}$ and $\boldsymbol{M}_{O \bar{B}}$ :

$$
\begin{aligned}
& \boldsymbol{M}_{T \bar{B}}=\boldsymbol{M}_{T O} \boldsymbol{M}_{O \bar{B}} \\
& =\boldsymbol{M}_{y}\left(\gamma_{T}\right) \boldsymbol{M}_{z}\left(-\Delta \psi_{T}\right) \boldsymbol{M}_{y}(-\theta),\left(\Delta \psi_{T} \triangleq \psi-\chi_{T}\right) \\
& =\left[\begin{array}{ccc}
c \gamma_{T} & 0 & -s \gamma_{T} \\
0 & 1 & 0 \\
s \gamma_{T} & 0 & c \gamma_{T}
\end{array}\right]\left[\begin{array}{ccc}
c \Delta \psi_{T} & -s \Delta \psi_{T} & 0 \\
s \Delta \psi_{T} & c \Delta \psi_{T} & 0 \\
0 & 0 & 1
\end{array}\right]\left[\begin{array}{ccc}
c \theta & 0 & s \theta \\
0 & 1 & 0 \\
-s \theta & 0 & c \theta
\end{array}\right] \\
& =\left[\begin{array}{ccc}
c \Delta \psi_{T} c \theta c \gamma_{T}+s \theta s \gamma_{T} & -s \Delta \psi_{T} c \gamma_{T} & c \Delta \psi_{T} s \theta c \gamma_{T}-c \theta s \gamma_{T} \\
s \Delta \psi_{T} c \theta & c \Delta \psi_{T} & s \Delta \psi_{T} s \theta \\
c \Delta \psi_{T} c \theta s \gamma_{T}-s \theta c \gamma_{T} & -s \Delta \psi_{T} s \gamma_{T} & c \Delta \psi_{T} s \theta s \gamma_{T}+c \theta c \gamma_{T}
\end{array}\right]
\end{aligned}
$$

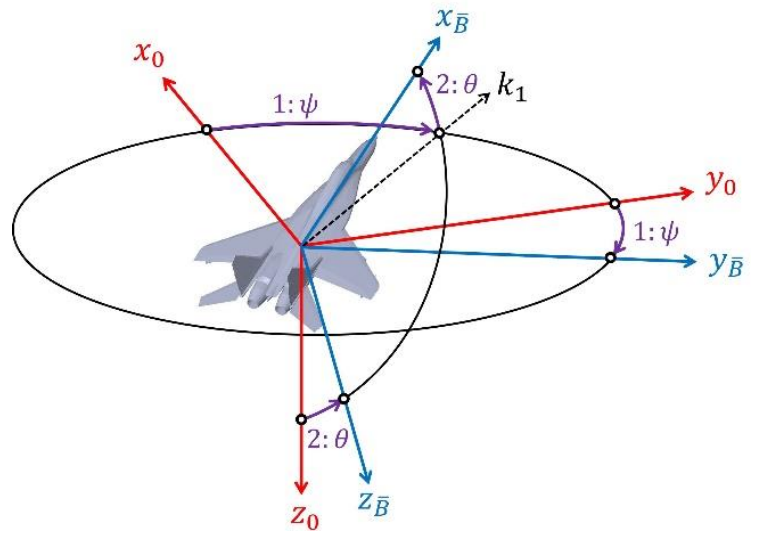

Fig. 21 Transformation from frame $\{O\}$ to frame $\{\bar{B}\}$

\section{Rigid Body Angular Velocity and Euler Angular Velocity}

The roll, pitch, yaw rates $P_{S}, Q_{S}, R_{S}$ around an aircraft's stability axis frame $\{S\}$ can be calculated via transforming the conventional roll, pitch, yaw rates $P, Q, R$ around body frame $\{B\}$ : 


$$
\left[\begin{array}{l}
P_{S} \\
Q_{S} \\
R_{S}
\end{array}\right]=M_{S B}\left[\begin{array}{l}
P \\
Q \\
R
\end{array}\right]=\left[\begin{array}{c}
P c \alpha+R s \alpha \\
Q \\
-P s \alpha+R c \alpha
\end{array}\right], M_{B S}=\left[\begin{array}{ccc}
c \alpha & 0 & -s \alpha \\
0 & 1 & 0 \\
s \alpha & 0 & c \alpha
\end{array}\right]
$$

The transformation relation between rigid body angular velocities and Euler angular velocities exists:

$$
\left[\begin{array}{l}
P \\
Q \\
R
\end{array}\right]=M_{x}(\phi)\left(M_{y}(\theta)\left[\begin{array}{l}
0 \\
0 \\
\dot{\psi}
\end{array}\right]+\left[\begin{array}{l}
0 \\
\dot{\theta} \\
0
\end{array}\right]\right)+\left[\begin{array}{l}
\dot{\phi} \\
0 \\
0
\end{array}\right]=\left[\begin{array}{ccc}
1 & 0 & -s \theta \\
0 & c \phi & s \phi c \theta \\
0 & -s \phi & c \phi c \theta
\end{array}\right]\left[\begin{array}{c}
\dot{\phi} \\
\dot{\theta} \\
\dot{\psi}
\end{array}\right]
$$

Solve for the above Euler angular velocity equation yields the following differential equation:

$$
\left[\begin{array}{c}
\dot{\phi} \\
\dot{\theta} \\
\dot{\psi}
\end{array}\right]=\left[\begin{array}{ccc}
1 & s \phi \tan \theta & c \phi \tan \theta \\
0 & c \phi & -s \phi \\
0 & s \phi / c \theta & c \phi / c \theta
\end{array}\right]\left[\begin{array}{l}
P \\
Q \\
R
\end{array}\right]
$$

